\title{
Study confirms certain risk factors for development of alveolar osteitis
}

\author{
Abstracted from \\ Halabí D, Escobar J, Muñoz C, Uribe S. \\ Logistic regression analysis of risk factors for the development of alveolar osteitis. \\ J Oral Maxillofac Surg 2012 May; 70: 1040-1044. doi: 10.1016/j.joms.2011.11.024. \\ Epub 2012 Feb 4. PubMed PMID: 22305872. \\ Address for correspondence: Dr S Uribe, School of Dentistry, Faculty of Medicine, \\ Universidad Austral de Chile, Rudloff 1640, Valdivia, Chile. E-mail: sergiouribe@uach.cl
}

\section{Question: What are the risk factors for alveolar osteitis?}

Design Prospective nested case-control study. Case/control selection From a cohort of 1423 Patents age 18 and older undergoing dental extractions at a public dental clinic in Chile examined at four days post-extraction. Eighty cases of alveolar osteitis were identified. Eighty matched controls were selected from the cohort at random.

Data analysis Eight predictor variables (risk factors), namely patient gender, hygiene, tooth location, previous surgical site infection, traumatic extraction, systemic diseases, alcohol consumption and tobacco use were considered. A binary regression logistic analysis was performed to determine significant associations.

Results The overall incidence of alveolar osteitis was 6.14\%. Logistic regression analysis after adjustment showed a statistically significant association between three variables and the development of alveolar osteitis; previous surgical site infection (odds ratio [OR], 3.3; $95 \% \mathrm{Cl}$, 1.4 to 7.7$)$, traumatic extraction (OR, $13.1 ; 95 \% \mathrm{Cl}, 5.4$ to 31.7 ) and smoking of tobacco after extraction (OR, 3.5; $95 \% \mathrm{Cl}, 1.3$ to 9.0 ). Conclusions Previous surgical site infection, traumatic extraction and tobacco smoking are associated with an increased risk of alveolar osteitis.

\section{Commentary}

Alveolar Osteitis, also known as dry socket, is a common post-operative complication and it has been an ongoing concern of dentists worldwide. It commonly occurs two to four days post-operatively, resulting in severe pain and discomfort for the patient. The aetiology is still vaguely understood and remains the subject of study. Some believe it may be related to bacteria that cause breakdown of the clot, or maybe the result of a traumatic extraction. Others studies have suggested a possible increased incidence in women who take oral contraceptives, due to estrogen. The lack of objective clinical criteria makes research difficult.

A nested case control study using a logistic regression model was used as the study design. A nested control study is generally more effective than a case-cohort design by matching factors and select- ing controls from relevant risk sets. In this study eight of the most commonly considered risk factors were selected for analysis. The study found that three out of eight of the risk factors demonstrated statistically significant results and concluded that there is an increased risk associated between previous surgical site infection, traumatic extraction and tobacco smoking for alveolar osteitis.

The sample size was derived from an original cohort of patients seeking emergency care within a 93-day period. Eighty incident cases were recruited. The sample size of 80 was determined based on the occurrence rate of 10 events per risk factor. An equal number of matched participants, selected at random, served as the control group.

Confounding factors accounted for were the number of cigarettes smoked per day. After a literature search the authors concluded that five or more cigarettes a day was considered a risk factor. Patient compliance was also mentioned. Postoperative care could not be followed or assessed. Another factor not mentioned, but that may introduce bias, was operator technique. Six surgeons were selected. It is uncertain how many patients were seen by each, as well as surgical techniques specific to each surgeon. These factors may influence outcomes. The authors provided sufficient data such as $\mathrm{p}$ values and confidence intervals, to assess the range and the magnitude of the effect.

Overall, this was a well conducted study, which further confirms the association of certain risk factors for the common and unwanted side effects of alveolar osteitis/dry socket.

Although previous studies have shown similar conclusions the results have applicable value to practitioners and patients.

\section{Practice point}

- Dentists should advise smokers that there is an increased risk of developing dry socket after an extraction in tobacco users. Practitioners should be aware that traumatic extractions or a previous history of surgical site infection could increase the chances of developing alveolar osteitis.

\section{Marie A Congiusta and Analia Veitz-Keenan} NYU College of Dentistry, New York, USA

Evidence-Based Dentistry (2013) 14, 86. doi:10.1038/sj.ebd.6400954 